EPJ manuscript No.

(will be inserted by the editor)

\title{
Internal structure of a quantum soliton and classical excitations due to trap opening
}

\author{
Yvan Castin \\ Laboratoire Kastler Brossel, École Normale Supérieure, UPMC and CNRS, 24 rue Lhomond, 75231 Paris Cedex 05, France
}

Received: 14 July 2008 / Revised version: 19 September 2008

\begin{abstract}
We analytically solve two problems that may be useful in the context of the recent observation of matter wave bright solitons in a one-dimensional attractive atomic Bose gas. The first problem is strictly beyond mean field: From the Bethe ansatz solution we extract the internal correlation function of the particle positions in the quantum soliton, that is for a fixed center of mass position. The second problem is solved in the limit of a large number of particles, where the mean field theory is asymptotically correct: It deals with the number of excitations created by the opening of the trap, starting from a pure soliton in a weakly curved harmonic potential.
\end{abstract}

PACS. 03.75.Lm Tunneling, Josephson effect, Bose-Einstein condensates in periodic potentials, solitons, vortices, and topological excitations - 03.75.Hh Static properties of condensates; thermodynamical, statistical, and structural properties - 03.75.Kk Dynamic properties of condensates; collective and hydrodynamic excitations, superfluid flow

Experiments with cold atoms have now acquired a high degree of control of the key parameters of the system. Using transverse confinement of the atoms by non-dissipative optical potentials it is possible to freeze the atomic motion along one or several directions, realizing in this way quantum gases with reduced dimensionality [1]. Furthermore, thanks to Feshbach resonances driven by a magnetic field, one can adjust almost at will the interaction strength between the atoms 2 . The combination of these two experimental tools has recently allowed the observation of bright solitons in a one-dimensional (1D) Bose gas, either a single soliton [3] or a train of solitons [4].

This leads to a renewed interest [5,6,7] in the 1D nonrelativistic Bose gas on the free line with zero range attractive interactions, that is with a binary interaction potential modeled by a Dirac delta with a negative coupling constant $g, V\left(x_{1}-x_{2}\right)=g \delta\left(x_{1}-x_{2}\right)$, a model that is an acceptable approximation of reality under conditions defined in 8 . On a theoretical point of view, it is well known that eigenstates and eigenenergies of the corresponding $N$ body Hamiltonian may be obtained by the Bethe ansatz: Historically the focus was put mainly on the study of the ground state wavefunction $\phi\left(x_{1}, \ldots, x_{N}\right)$ [9, 10, which is a collective bound state of the $N$ particles, the so-called $N$-particle quantum soliton, with a delocalized center of mass of vanishing momentum. The extension of the Bethe ansatz to excited states is however possible, and one finds that the generic excited state corresponds to a set of quantum solitons with arbitrary atom numbers and different momenta per particle [11. A key property to keep in mind is the full separability of the center of mass variables and the internal variables of the gas (e.g. the relative coordinates of the particles), which holds since the gas is on the free line with open boundary conditions.

Despite the knowledge of the ground state wavefunction for $N$ particles, some theoretical work is needed to extract experimentally relevant observables. As atomic density profiles may be measured by absorption or even nondestructive imaging [12, natural observables are functions of the positions of the particles. The simplest observable is the mean density of particles, $\rho(x)$, obtained by an average of the density profile over many (ideally infinitely many) experimental realizations. From the ground state $N$-body wavefunction one however does not obtain any useful information on the mean density: Since the center of mass is fully delocalized, one gets a uniform distribution over the whole line. Experimental reality is very different, the soliton being obtained from an initially trapped BoseEinstein condensate. When the trap is switched off to free the soliton, its center of mass is not in its ground state, it is in a localized and non-stationary state which depends on the experimental preparation procedure. A more realistic assumption is thus to assume a $N$-body wavefunction of the form

$$
\Psi\left(x_{1}, \ldots, x_{N}\right)=\Phi(R) \phi\left(x_{1}, \ldots, x_{N}\right),
$$

where

$$
R=\frac{1}{N} \sum_{i=1}^{N} x_{i}
$$

is the center of mass position of the soliton and the center of mass wavefunction $\Phi(R)$ is a priori unknown and de- 
Yvan Castin: Internal structure of a quantum soliton and classical excitations due to trap opening

pends on the experimental details. The theoretical challenge is thus to predict observables for a fixed position of the center of mass $R$. Experimentally relevant results are then obtained from these theoretical predictions by a further average over $R$ with the probability distribution $|\Phi(R)|^{2}$. One can even hope that the predictions for fixed $R$ are measurable, if the center of mass position can be measured with a high enough accuracy for each individual realization of the experiment.

Turning back to the simple example of the mean density, we see that the right concept is the mean density of particles $\rho(x \mid R)$ for a fixed center of mass position $R$, as was already argued in [11] within the general concept of (here translational) symmetry breaking. Remarkably an explicit expression of $\rho(x \mid R)$ in terms of a sum of $N-1$ exponential terms may be obtained [13.14.

The next step beyond the mean density is the pair distribution function of the particles $\rho(x, y)$, or very similarly the static structure factor $S(x, y)$, of the $N$-particle soliton. Recently a general study of the dynamic structure factor was performed from the Bethe ansatz [6,7], which includes the static structure factor $S(x, y)$ and large $N$ expansions as limiting cases. The goal of the present work is, in the spirit of the above physical discussion, to study the static structure factor $S(x, y \mid R)$ for a fixed center of mass position $R$. This static structure factor gives access to correlations between the positions of the particles inside the soliton, that is it gives information on the internal structure of the soliton, which goes beyond the usual mean field (or Gross-Pitaevskii) approximation, which neglects such correlations.

As a guideline we imagine that, in an experiment, one wishes to access the variance of a one-body observable of the gas involving some function $U(x)$ of the particle position $x$ :

$$
w(R)=\left\langle\left[\sum_{i=1}^{N} U\left(x_{i}\right)\right]^{2}\right\rangle_{R}-\left\langle\sum_{i=1}^{N} U\left(x_{i}\right)\right\rangle_{R}^{2}
$$

where the expectation value $\langle\ldots\rangle_{R}$ is taken over the internal wavefunction $\phi\left(x_{1}, \ldots, x_{N}\right)$ of the quantum soliton for a fixed center of mass location $R$.

The paper is organized as follows. In section 1 we recall the basic facts about the 1D Bose gas model and we give a general expression of the pair distribution function $\rho(x, y \mid R)$ for fixed $R$ that may be used to evaluate $w(R)$ numerically for a moderate number of atoms and that will be the starting point of analytical calculations. In section 2 we present an analytical calculation of $w(R)$ for an arbitrary atom number $N$, in the limit where the function $U(x)$ is slowly varying over the spatial width of the soliton. In section 3 we present a large $N$ expansion of the static structure factor $S(x, y \mid R)$ for fixed $R$ that we then use to calculate $w(R)$ to leading order in $N$; in the same section, we integrate $S(x, y \mid R)$ over $R$ to see if we recover the results of [7] for $S(x, y)$, and we also consider the case of a function $U(x)$ much narrower than the soliton. In section 4 we evaluate the accuracy of the assumption (11) in an experiment: Assuming that the gas in the trap is in its ground state (at least for its internal variables), we calculate analytically (to leading order in $N$ and in the trap curvature) the number of internal excitations of the soliton produced by the trap opening. We conclude in section 5.

\section{Model, basic definitions and general results}

\subsection{Hamiltonian and ground state wavefunction}

We consider a set of $N \geq 2$ spinless non-relativistic bosons of mass $m$ moving on the one dimensional real line, in the absence of any trapping potential, with open boundary conditions, and binary interacting via an attractive contact potential of coupling constant $g<0$. This corresponds to the Hamiltonian in first quantized form:

$$
H=\sum_{i=1}^{N}-\frac{\hbar^{2}}{2 m} \partial_{x_{i}}^{2}+\sum_{1 \leq i<j \leq N} g \delta\left(x_{i}-x_{j}\right) .
$$

For this problem there is full separability of the center of mass motion and of the internal coordinates, the internal wavefunction being independent of the center of mass state. As a consequence, the ground state wavefunction has it center of mass with zero momentum and depends only on the relative coordinates of the particles. Its exact expression is 9 ]

$$
\phi\left(x_{1}, \ldots, x_{N}\right)=\mathcal{N} \exp \left[-\frac{m|g|}{2 \hbar^{2}} \sum_{1 \leq i<j \leq N}\left|x_{i}-x_{j}\right|\right]
$$

where the normalization factor $\mathcal{N}$ will be specified later. The corresponding eigenenergy is [9]

$$
E_{0}(N)=-\frac{m g^{2}}{24 \hbar^{2}}(N-1) N(N+1)
$$

This ground state is the so-called quantum soliton with $N$ particles.

\subsection{Mean density, pair distribution and static structure factor for a fixed center of mass position}

The crucial concept in the present work is the expectation value of a position dependent observable, that is of an arbitrary function $O\left(x_{1}, \ldots, x_{N}\right)$ of the $N$ particle positions, for a fixed value $R$ of the center of mass position:

$$
\begin{aligned}
\langle O\rangle_{R} \equiv & \int d x_{1} \ldots d x_{N} \delta\left(R-\frac{1}{N} \sum_{k=1}^{N} x_{k}\right) \\
& \times O\left(x_{1}, \ldots, x_{N}\right)\left|\phi\left(x_{1}, \ldots, x_{N}\right)\right|^{2}
\end{aligned}
$$

where the integration is over the whole space $\mathbb{R}^{N}$. The soliton wavefunction shall then be normalized in such a way that the expectation value $\langle 1\rangle_{R}$ of the function $O$ constant and equal to unity is also equal to unity. Using 
the bosonic symmetry of the wavefunction, the integral to compute is then simply $N$ ! times the integral over the so-called fundamental domain of ordered positions

$$
D=\left(x_{1}, \ldots, x_{N}\right) \text { such that } x_{1}<\ldots<x_{N} .
$$

Over this domain, the wavefunction indeed has the separable expression

$$
\phi\left(x_{1}, \ldots, x_{N}\right)=\mathcal{N} e^{-\frac{m|g|}{2 \hbar^{2}} \sum_{j=1}^{N}[2 j-(N+1)] x_{j}} .
$$

With the change of variables (94) one then obtains

$$
|\mathcal{N}|^{2}\left(\frac{\hbar^{2}}{m|g|}\right)^{N-1} \frac{N}{(N-1) !}=1 .
$$

Setting $O=\sum_{j} \delta\left(x-x_{j}\right)$ we obtain the mean density $\rho(x \mid R)$ for a fixed center of mass position. This was first calculated in [13]:

$$
\rho(x \mid R)=\frac{N !^{2}}{N \xi} \sum_{k=0}^{N-2} \frac{(-1)^{k}(k+1)}{(N-2-k) !(N+k) !} e^{-(k+1)|x-R| / \xi},
$$

where $\xi$ is the spatial width of the classical (that is meanfield) soliton,

$$
\xi=\frac{\hbar^{2}}{m|g| N}
$$

It is a function of $|x-R|$, a consequence of translational and parity invariance of the Hamiltonian and of $\phi$. One thus has $\rho(x \mid R)=\rho(x-R \mid 0)=\rho(R-x \mid 0)$. It is normalized in such a way that the integral over $x$ over the whole space is equal to $N$. If the true physical state of the system is a product (1) of a localized center of mass wavefunction $\Phi(R)$ and of the quantum soliton wavefunction, the physical mean density is the average (or equivalently the convolution)

$$
\rho(x)=\int_{-\infty}^{+\infty} d R|\Phi(R)|^{2} \rho(x-R \mid 0) .
$$

Setting $O=\sum_{i \neq j} \delta\left(x-x_{i}\right) \delta\left(y-x_{j}\right)$, we obtain the pair distribution function $\rho(x, y \mid R)$ for fixed center of mass position $R$. It is normalized as $N(N-1)$ for the double integration over $x$ and $y$. From the translation invariance $\rho(x, y \mid R)=\rho(x-R, y-R \mid 0)$ so that it is sufficient to calculate this pair distribution function for $R=0$. In real space, we do not know an expression of $\rho(x, y \mid 0)$ as simple as (11) but we have found a simple expression for the Fourier transform

$$
\tilde{\rho}\left(q_{a}, q_{b} \mid 0\right) \equiv \int_{\mathbb{R}^{2}} d x d y \rho(x, y \mid 0) e^{-i\left(q_{a} x+q_{b} y\right)} .
$$

As detailed in the appendix $\mathrm{A}$ one has

$$
\begin{gathered}
\tilde{\rho}\left(q_{a}, q_{b} \mid 0\right)=\left|\frac{\Gamma(N)}{\Gamma(N+i Q)}\right|^{2} \sum_{1 \leq j<k \leq N} \\
\frac{\Gamma(k-i Q) \Gamma(N+1+i Q-j)}{\Gamma(j) \Gamma(N+1-k)} \\
\times\left[\frac{\Gamma\left(j-\epsilon_{a}\right) \Gamma\left(N+1+i Q-\epsilon_{a}-k\right)}{\Gamma\left(k-\epsilon_{a}\right) \Gamma\left(N+1+i Q-\epsilon_{a}-j\right)}+\epsilon_{a} \leftrightarrow \epsilon_{b}\right]
\end{gathered}
$$

where $\Gamma(z)$ is the Gamma function of complex argument $z$ and we have introduced the dimensionless variables $Q_{a, b}=$ $q_{a, b} \xi, Q=Q_{a}+Q_{b}$ and $q=\left(Q_{b}-Q_{a}\right) / 2$. The quantities $\epsilon_{a, b}$ solve the degree two equations:

$$
\epsilon_{a, b}\left(N+i Q-\epsilon_{a, b}\right)=i Q_{a, b} N
$$

In the large $N$ limit, for fixed values of $Q_{a}$ and $Q_{b}$, it is convenient to take

$$
\epsilon_{a, b}=\frac{1}{2}(N+i Q)-\left[(i q \pm N / 2)^{2}-Q_{a} Q_{b}\right]^{1 / 2}
$$

with a determination of the square root such that $\epsilon_{a, b}=$ $i Q_{a, b}+Q_{a} Q_{b} / N+O\left(1 / N^{2}\right)$ for $N \rightarrow+\infty$, e.g. with the line cut on the real negative axis.

This gives the idea a posteriori to look for a similar expression for the Fourier transform $\tilde{\rho}\left(q_{a} \mid 0\right)$ of $\rho(x \mid 0)$. As shown in the appendix $\mathrm{A}$ one obtains the simple expression

$$
\begin{aligned}
\tilde{\rho}\left(q_{a} \mid 0\right)= & \left|\frac{\Gamma(N)}{\Gamma\left(N+i Q_{a}\right)}\right|^{2} \\
& \times \sum_{j=1}^{N} \frac{\Gamma\left(j-i Q_{a}\right) \Gamma\left(N+1+i Q_{a}-j\right)}{\Gamma(j) \Gamma(N+1-j)} .
\end{aligned}
$$

Amusingly, this allows to express $\tilde{\rho}\left(q_{a} \mid 0\right)$ in terms of the standard hypergeometric function evaluated in $z=1$,

$$
\begin{aligned}
\tilde{\rho}\left(q_{a} \mid 0\right)=\frac{\Gamma(N) \Gamma\left(1-i Q_{a}\right)}{\Gamma\left(N-i Q_{a}\right)} \times & \\
& { }_{2} F_{1}\left(1-N, 1-i Q_{a} ; 1-N-i Q_{a} ; 1\right),
\end{aligned}
$$

although we have not found this expression particularly useful.

Let us come back to the original problem of calculating the variance $w(R)$ of the one-body observable $\sum_{i} U\left(x_{i}\right)$, as defined in (3). One first expresses the squares as products of double sums over indices $i$ and $j$. One can split the double sum over $i$ and $j$ in (3) in diagonal terms $i=j$ and off-diagonal terms $i \neq j$, so that $w(R)=w_{\text {diag }}(R)+$ $w_{\text {off }}(R)$. The diagonal terms can be expressed in terms of the mean density,

$$
\begin{gathered}
w_{\text {diag }}(R) \equiv \sum_{i=1}^{N}\left\langle U\left(x_{i}\right)^{2}\right\rangle_{R}-\left[\sum_{j=1}^{N}\left\langle U\left(x_{j}\right)\right\rangle_{R}\right]^{2} \\
=\int_{\mathbb{R}} d x \rho(x \mid R) U(x)^{2}-\left[\int_{\mathbb{R}} d x \rho(x \mid R) U(x)\right]^{2} .
\end{gathered}
$$

This can be directly evaluated from (11). The off-diagonal terms depend on the pair distribution function,

$$
\begin{aligned}
w_{\mathrm{off}}(R)=\sum_{i \neq j}\left\langle U\left(x_{i}\right) U\left(x_{j}\right)\right\rangle_{R} & \\
= & \int_{\mathbb{R}^{2}} d x d y U(x) U(y) \rho(x, y \mid R) .
\end{aligned}
$$


Introducing the Fourier transform of $U(x)$,

$$
\tilde{U}\left(q_{a}\right)=\int_{\mathbb{R}} d x U(x) \exp (-i q x),
$$

we obtain the Fourier space expression

$$
\begin{aligned}
w_{\text {off }}(R)=\int_{\mathbb{R}^{2}} \frac{d q_{a} d q_{b}}{(2 \pi)^{2}} \tilde{U}\left(q_{a}\right) \tilde{U}\left(q_{b}\right) \\
\\
\times e^{i\left(q_{a}+q_{b}\right) R} \tilde{\rho}\left(-q_{a},-q_{b} \mid 0\right)
\end{aligned}
$$

that can be directly evaluated using (15). Simpler analytic expressions of $w(R)$, either for slowly varying functions $U(x)$ or in the large $N$ limit, shall be given in the next sections.

To conclude this subsection, we note that $w(R)$ has a very simple expression in terms of the correlations contained in the fixed $R$ static structure factor $S(x, y \mid R)$,

$$
\begin{aligned}
& S(x, y \mid R) \equiv\langle\hat{\rho}(x) \hat{\rho}(y)\rangle_{R} \\
& \quad=\delta(x-y) \rho(x \mid R)+\rho(x, y \mid R)
\end{aligned}
$$

where the operator giving the density is

$$
\hat{\rho}(x)=\sum_{i=1}^{N} \delta\left(x_{i}-x\right)
$$

We note that the double integral of $S(x, y)$ over $x$ and $y$ is equal to $N^{2}$. Using again the translational invariance, one obtains the illuminating expression

$$
\begin{aligned}
w(R)=\int_{\mathbb{R}^{2}} d x d y U & (x+R) U(y+R) \\
\times & {[S(x, y \mid 0)-\rho(x \mid 0) \rho(y \mid 0)] . }
\end{aligned}
$$

The essence of the mean-field approximation is to neglect correlations among the particles, so that $S(x, y \mid 0)$ would essentially be approximated by the uncorrelated product $\rho(x \mid 0) \rho(y \mid 0)$. The above writing clearly reveals that $w(R)$ is sensitive to correlations that are beyond the mean-field approximation.

\subsection{The usual static structure factor}

The usual static structure factor is the spatial correlation function of the operator $\hat{\rho}(x)$ giving the density,

$$
S(x, y)=\langle\hat{\rho}(x) \hat{\rho}(y)\rangle
$$

where the expectation value is taken literally in the ground state of the gas, thus assuming a perfectly delocalized center of mass wavefunction $\Phi(R)=1$. Because of the translational invariance, it is a function of $x-y$ only.

This usual structure factor is deduced from our fixed- $R$ one by integration over the center of mass position

$$
S(x, y)=\int_{\mathbb{R}} d R S(x, y \mid R)=\int_{\mathbb{R}} d R S(x-R, y-R \mid 0) .
$$

This allows to express the Fourier transform of $S(x, y)$ in terms of the Fourier transform of $\rho(x, y \mid 0)$, when one uses (24):

$$
\tilde{S}\left(q_{a}, q_{b}\right)=2 \pi \delta\left(q_{a}+q_{b}\right)\left[N+\tilde{\rho}\left(q_{a}, q_{b} \mid 0\right)\right] .
$$

From (15) we thus have an analytical expression of $\tilde{S}\left(q_{a}, q_{b}\right)$ in terms of a double sum.

The relation (28) will also allow us, in the large $N$ limit, to convert our large $N$ expansion of $S(x, y \mid R)$ into a large $N$ expansion of $S(x, y)$, see 33.2 .

\section{Value of $w(R)$ for a broad function $U(x)$}

One supposes in this section that $U(x)$ varies slowly over the length scale $\xi$ of the quantum soliton, as defined in (12). Then one rewrites (3) using the translational invariance,

$$
w(R)=\left\langle\left[\sum_{i=1}^{N} U\left(x_{i}+R\right)\right]^{2}\right\rangle_{0}-\left\langle\sum_{i=1}^{N} U\left(x_{i}+R\right)\right\rangle_{0}^{2},
$$

and one expands

$$
U\left(x_{i}+R\right)=U(R)+x_{i} U^{\prime}(R)+\frac{1}{2} x_{i}^{2} U^{\prime \prime}(R)+\ldots
$$

The constant shift $U(R)$ has no effect in $w(R)$. The linear term has also an exactly vanishing contribution, since by definition $\left\langle\left(\sum_{i=1}^{N} x_{i}\right)^{n}\right\rangle_{0}=0$ for all integers $n \geq 1$. Setting

$$
O_{2}=\sum_{i=1}^{N} x_{i}^{2}
$$

we thus obtain the leading contribution

$$
w(R) \simeq \frac{1}{4}\left[U^{\prime \prime}(R)\right]^{2}\left(\operatorname{Var} O_{2}\right)_{0},
$$

with $\left(\operatorname{Var} O_{2}\right)_{0}=\left\langle O_{2}^{2}\right\rangle_{0}-\left\langle O_{2}\right\rangle_{0}^{2}$ is the variance of $O_{2}$ for a center of mass position fixed at the origin of the coordinates.

It turns out that an exact expression may be obtained for this variance, as detailed in the appendix $B$, It is the sum of three contributions,

$$
\left(\operatorname{Var} O_{2}\right)_{0}=\left(\frac{\hbar^{2}}{m|g|}\right)^{4}\left[S_{1}+S_{2}+S_{3}\right],
$$

with

$$
\begin{aligned}
& S_{1}=4 \sum_{i=2}^{N} \sum_{j=2}^{N} \sum_{k=2}^{N} B_{i j} B_{i k} \\
& S_{2}=\sum_{i=2}^{N} \sum_{j=2}^{N}\left[8 B_{i i} B_{i j}+2 B_{i j}^{2}\right] \\
& S_{3}=6 \sum_{i=2}^{N} B_{i i}^{2} .
\end{aligned}
$$


We have introduced the symmetric matrix

$$
B_{i j}=\frac{1}{N} \frac{1}{[N+1-\min (i, j)][\max (i, j)-1]},
$$

defined over the index range $2 \leq i, j \leq N$. This holds whatever the value of the atom number $N \geq 2$.

In the large $N$ limit, the contribution $S_{1}$ is dominant, simply because it contains more terms, and its asymptotic expression is evaluated by replacing the sums by integrals,

$$
\begin{aligned}
S_{1} \simeq \frac{4}{N^{3}} \int_{0}^{1} d x\left[\frac{\ln (1-x)}{x}\right. & \left.+\frac{\ln x}{1-x}\right]^{2} \\
= & N^{-3}\left[\frac{8 \pi^{2}}{3}+16 \zeta(3)\right] .
\end{aligned}
$$

This leads to the estimate

$$
w(R) \simeq N \xi^{4}\left[U^{\prime \prime}(R)\right]^{2}\left[\frac{2 \pi^{2}}{3}+4 \zeta(3)\right],
$$

for a slowly varying potential $U(x)$ in the $N \gg 1$ limit.

\section{Large $N$ limit of $w(R)$ for $U(x)$ of any width}

In this section we give a large $N$ expansion of the static structure factor for fixed center of mass position, which allows to get the asymptotic behavior of $w(R)$ in the large $N$ limit. When specialized to a quadratic potential $U(x)$ the general result reproduces the large $N$ broad potential result of the previous section. As a first test of the result, we integrate $S(x, y \mid R)$ over the center of mass position $R$, to see if we recover the results of [7] for the usual static factor $S(x, y)$. As a second test of the result, we get an approximate expression for $w(R)$ in the case of a narrow function $U(x)$, to lowest order in the width $b$ of $U(x)$, first from the large $N$ expansion of $S(x, y \mid R)$ and then from a more general reasoning not relying on a large $N$ expansion.

\subsection{Asymptotic expression of $S(x, y \mid R)$ and of $w(R)$}

As we have seen in (26), $w(R)$ is directly related to the deviation of the fixed $R$ static structure factor of the soliton from the uncorrelated form $\rho(x \mid R) \rho(y \mid R)$. It turns out that this deviation may be easily obtained from the Fourier space expressions (15]18), simply by taking the large $N$ limit of the $\Gamma$ functions and by replacing the discrete sums over indices by integrals. This shows that our Fourier space representations are indeed useful.

As detailed in the appendix $\mathrm{C}$ the large $N$ expansion for a fixed value of $\xi$ (that is for a fixed value of $\mathrm{Ng}$ ), gives the leading term

$$
\begin{aligned}
& S(x, y \mid 0)-\rho(x \mid 0) \rho(y \mid 0) \simeq-N \partial_{x} \partial_{y} \\
& {\left[\theta(y-x) \frac{y / \xi-x / \xi-e^{x / \xi}-e^{-y / \xi}}{[2 \cosh (x / 2 \xi)]^{2}[2 \cosh (y / 2 \xi)]^{2}}+x \leftrightarrow y\right]}
\end{aligned}
$$

where $\theta(x)$ is the Heaviside distribution that is equal to zero for $x<0$ and to one for $x>0$. If one wishes to have an expansion of the static structure factor only, one has also to expand $\rho(x \mid 0)$ in powers of $N$. From the real space expression (11), expanding each factorial in the large $N$ limit for a fixed summation index $k$, we obtain, setting $X=x / \xi$, a result in agreement with [13]:

$$
\rho(x \mid 0)=\frac{N}{\xi}\left[1-\frac{1}{N} \frac{d^{2}}{d X^{2}}+\ldots\right] \frac{1}{[2 \cosh (X / 2)]^{2}},
$$

where one may check the normalization condition $N=$ $\int_{\mathbb{R}} d x \rho(x \mid 0)$. This gives the expansion of $S(x, y \mid 0)$ up to order $N$, for a fixed value of $\xi$.

From (24) and (41) we can directly obtain, in the large $N$ limit, the pair correlations between the positions of the particles for a fixed center of mass position:

$$
\delta \rho(x, y \mid R) \equiv \rho(x, y \mid R)-\rho(x \mid R) \rho(y \mid R) .
$$

One can indeed show that the distributions generated in (411) by the derivatives of $\theta(x-y)$ and $\theta(y-x)$ with respect to $x$ and $y$ exactly cancel with the Dirac term appearing in (24). As a consequence the expression for $\delta \rho(x, y \mid 0)$ is deduced from the right hand side of (41) simply by exchanging the order of the $\theta$ distributions and of the operator $\partial_{x} \partial_{y}$. After an explicit calculation of the derivatives with respect to $x$ and $y$ we obtain

$$
\begin{gathered}
\delta \rho(x, y \mid 0) \simeq-\frac{N}{16 \xi^{2}}[(2+|X-Y|) \sinh (X / 2) \sinh (Y / 2) \\
\quad+2 \sinh (|X-Y| / 2)] /[\cosh (X / 2) \cosh (Y / 2)]^{3}, \quad(44)
\end{gathered}
$$

with $X=x / \xi$ and $Y=y / \xi$. A contour plot of this function reveals that it has an interesting structure, in the form of two valleys separated by a crest on the $x=y$ line, each valley being elongated in the direction parallel to $x=y$ and containing two local minima separated by a saddle point. For clarity we only show a plot of $\delta \rho$ along the line $y=-x$, restricting to $x>0$ by parity, see the thick solid line in Fig 1 the minimum of this line then corresponds in the full $x-y$ plane to one of the aforementioned saddle points. In the same figure, we also give the value of $\delta \rho$ for finite values of $N$, obtained by calculating the Fourier transform of (101) and (103) numerically. This shows that the large $N$ limit is well approached with moderately high values of $N$ already.

We come back to (41) to obtain the large $N$ equivalent of $w(R)$ at fixed $\xi$. By repeated integration by parts in the double integral of (26), and using the fact that

$$
-\frac{Y-X-e^{X}-e^{-Y}}{[2 \cosh (X / 2)]^{2}[2 \cosh (Y / 2)]^{2}}=\partial_{X} \partial_{Y} \frac{2+Y-X}{\left(e^{Y}+1\right)\left(e^{-X}+1\right)},
$$

we find 15 for a function $U(x)$ not rapidly increasing at $|x|=\infty$ (that is not increasing faster than a power law):

$$
\begin{aligned}
& w(R) \simeq 2 N \xi^{4} \int_{-\infty}^{+\infty} d X \int_{X}^{+\infty} d Y \\
& U^{\prime \prime}(R+X \xi) U^{\prime \prime}(R+Y \xi) \frac{2+Y-X}{\left(e^{Y}+1\right)\left(e^{-X}+1\right)} .
\end{aligned}
$$




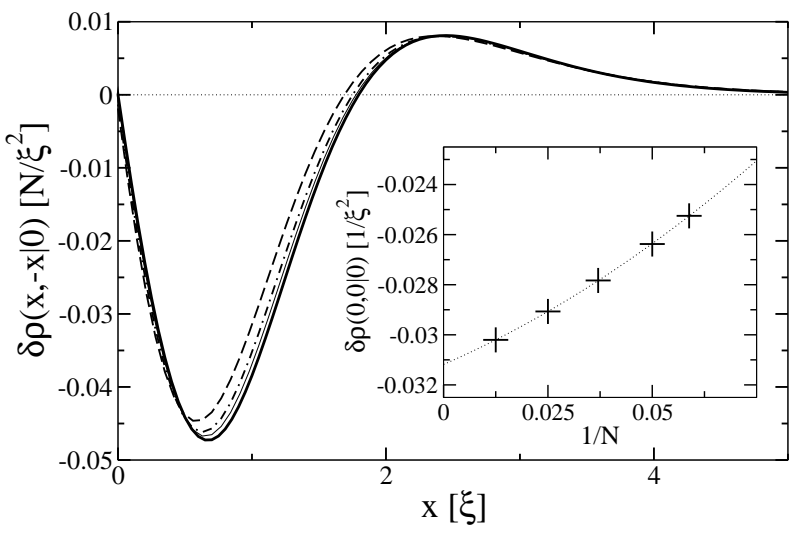

Fig. 1. Cut along the line $y=-x$ of the function $\delta \rho(x, y \mid 0)=$ $\rho(x, y \mid 0)-\rho(x \mid 0) \rho(y \mid 0)$. Thick solid line: Large $N$ limit (44). Dashed line: Numerical result for $N=10$. Dot-dashed line: Numerical result for $N=20$. Thin solid line: Numerical result for $N=40 . \delta \rho$ is in units of $N / \xi^{2}$, and the coordinate $x$ is in units of $\xi$. The inset shows numerical evidence for the convergence of $\delta \rho(0,0 \mid 0) \xi^{2}$ towards a non-zero value $\simeq-0.031$ at large $N$, which is compatible with the analytical prediction $\delta \rho(0,0 \mid 0) \xi^{2} / N \rightarrow 0$. In the inset, symbols are numerical data, and the dotted line is a quadratic fit used to guide the eye.

One immediately sees that a linearly varying potential $U(x)$ gives a vanishes contribution to $w(R)$, which is also obvious from the definition (3), since $\left\langle\left(\sum_{i} x_{i}\right)^{n}\right\rangle_{R}=$ $(N R)^{n}$ by construction. If $U(x)$ is a quadratic function of $x, U(x)=U^{\prime \prime}(R) x^{2} / 2$, one should recover Eq. (40), which is indeed the case if

$$
\frac{\pi^{2}}{3}+2 \zeta(3)=\int_{-\infty}^{+\infty} d X \int_{X}^{+\infty} d Y \frac{2+Y-X}{\left(e^{Y}+1\right)\left(e^{-X}+1\right)} .
$$

We have checked that this identity holds [16].

\subsection{Application: large $N$ expansion of the usual static structure factor}

The usual static structure factor $S(x, y)$ is obtained from $S(x, y \mid R)$ by integration over $R$, see (28). Assuming without loss of generality that $y=0$, we see from (41) and from (45) that one has to integrate over $R$ quantities of the form $\left(\partial_{x} \partial_{y} F\right)(x-R, R)$, where the function $F$ corresponds in a first stage to the expression in between square brackets in (41) and in a second stage to the function over which $\partial_{X} \partial_{Y}$ acts in the right hand side of (45). From the differential relations, taking $x$ and $R$ as independent variables,

$$
\begin{aligned}
& \left(\partial_{x} F\right)(x-R,-R)=\frac{d}{d x}[F(x-R,-R)] \\
& \left(\partial_{y} F\right)(x-R,-R)=-\left(\frac{d}{d x}+\frac{d}{d R}\right)[F(x-R,-R)],
\end{aligned}
$$

we see that the integral over $R$ will cancel the derivatives $d / d R$. Using

$$
\int_{-\infty}^{+\infty} d R \frac{1}{\left(e^{R}+1\right)\left(e^{-R+X}+1\right)}=\frac{X}{e^{X}-1}
$$

one gets

$$
\begin{aligned}
& S(x, 0)-\int_{-\infty}^{+\infty} d R \rho(x \mid R) \rho(0 \mid R) \simeq \\
& \frac{N}{\xi} \frac{d^{2}}{d X^{2}}\left[\theta(X) \frac{d^{2}}{d X^{2}} \frac{X(X+2)}{e^{X}-1}+X \leftrightarrow-X\right],
\end{aligned}
$$

where $X=x / \xi$. One can show that the $\theta(X)$ distribution can be exchanged with the last $d^{2} / d X^{2}$ if one wishes, but we shall not use this property here. From the large $N$ expansion (42) of $\rho(x \mid R)$, and using the fact that the second order derivative of $X /[\exp (X)-1]$ is an even function, one finally obtains the large $N$ expansion

$$
\begin{aligned}
& S(x, y=0)=\frac{N^{2}}{\xi} \frac{d^{2}}{d X^{2}}\left[\frac{X}{e^{X}-1}\right] \\
& +\frac{N}{\xi} \frac{d^{2}}{d X^{2}}\left[\theta(X) \frac{d^{2}}{d X^{2}} \frac{X^{2}}{e^{X}-1}+X \leftrightarrow-X\right]+\ldots . .
\end{aligned}
$$

To compare this result with the ones of [7] given in Fourier space, one performs the Fourier transform of Eq.(62) and Eq.(63) of [7. Then one finds that (52) is consistent with [7] if one adds in Eq.(62) of [7] the subleading term of the large $N$ expansion of the intermediate quantity $S_{N}^{\rho}(k)$ introduced in 7.

Furthermore, (52) can be shown to be equivalent to the more appealing writing, where the expected Dirac delta contribution is singled out,

$$
\begin{gathered}
S(x, y=0)=\frac{N^{2}}{\xi} \frac{d^{2}}{d X^{2}}\left[\frac{X / 2}{\tanh (X / 2)}\right]+N \delta(x) \\
+\frac{N}{\xi}\left\{\theta(X) \frac{d^{4}}{d X^{4}}\left[\frac{X^{2} / 2}{\tanh (X / 2)}\right]+X \leftrightarrow-X\right\}+\ldots
\end{gathered}
$$

where we recall that $X=x / \xi$. One can also easily check that this obeys the sum rule $\int_{\mathbb{R}} d x S(x, y=0)=N^{2}$.

\subsection{Approximation of $w(R)$ for a narrow barrier}

In this subsection, one assumes that $U(x)$ and its derivatives are functions localized around the origin with a width much smaller than $\xi$. E.g. $U(x)$ is a Gaussian centered in $x=0$ with a width $\ll \xi$. One first considers the large $N$ limit. We rewrite Eq. (46) as

$$
\begin{aligned}
w(R) \simeq 2 N \xi^{2} \int_{-\infty}^{+\infty} d x \int_{x}^{+\infty} d y & \\
& U^{\prime \prime}(x) U^{\prime \prime}(y) F\left(\frac{x-R}{\xi}, \frac{y-R}{\xi}\right)
\end{aligned}
$$

with

$$
F(X, Y)=\frac{2+Y-X}{\left(e^{Y}+1\right)\left(e^{-X}+1\right)} .
$$

Then we expand the factor containing $F$ in powers of $x / \xi$ and $y / \xi$. If $U(x)$ and its derivatives are rapidly decreasing 
functions, one can show that the first non vanishing contribution to $w(R)$ comes from the third order expansion, which results in

$$
w(R) \simeq \rho(R \mid 0) \int_{-\infty}^{+\infty} d x U(x)^{2}
$$

where we have used (42) to leading order in $N$ to recognize the factor $\rho(R \mid 0)$.

There is actually a faster way to obtain this result, and not restricted to the large $N$ limit, from the exact writing

$$
\begin{aligned}
w(R)=\int d x \rho(x \mid R) U(x)^{2}+\int_{\mathbb{R}^{2}} d x d y \\
\\
{[\rho(x, y \mid R)-\rho(x \mid R) \rho(y \mid R)] U(x) U(y) . }
\end{aligned}
$$

Then one sees that the first term in (57) is first order in the width $b$ of $U(x)$, since the integration range over $x$ has a width $b$, whereas the second term is second order in $b$, since it involves a double integral over a range of diameter $\sim b$. Then to first order in $b$ one recovers (56). In the large $N$ limit, for a fixed $\xi$, both terms of (57) scale in the same way with $N$, that is linearly with $N$, so that the order of the limits $N \rightarrow+\infty$ and $b \rightarrow 0$ (for fixed $\xi$ ) is not crucial.

\section{Number of internal excitations created by the trap opening}

In this work, we have assumed up to now that a pure soliton is produced in the experiment, corresponding to the state (1): The center of mass may be in an arbitrary excited state but the internal variables of the gas are in their ground state. In this section, we revisit this assumption taking into account experimental constraints.

In a real experiment the ultracold gas is prepared in a trap; it is not free along $x$, and each atom is subject to the harmonic confining potential

$$
W(x)=\frac{1}{2} m \omega^{2} x^{2} .
$$

We recall that the center of mass motion and the internal variables remain separable in a harmonic trap. In order for the trapped gas to be close to the free space limit, the oscillation frequency $\omega$ is adjusted to have $\hbar \omega \ll\left|\mu_{0}\right|$, where

$$
\mu_{0}=-\frac{\hbar^{2}}{8 m \xi^{2}}
$$

is the mean-field approximation for the chemical potential of the free-space soliton. We assume that the trapped gas is cooled down to a temperature $T$ low enough to have $k_{B} T \ll\left|\mu_{0}\right|$. The gas is then a pure soliton. On the contrary we do not assume the much more stringent condition $k_{B} T \ll \hbar \omega$, so that the center of mass of the gas may still be in an excited state.

Eventually the trapping potential along $x$ will be switched off, to obtain the ideal conditions of the Hamiltonian (4). This trap opening will create some internal excitations of the gas, that is the untrapped gas will not be a pure soliton but will contain a mean number $N_{\text {exc }}$ of internal excitations.

Since no Bethe ansatz solution exists in a trap, the modest goal here is to calculate $N_{\text {exc }}$ to leading order in $N$ in the large $N$ limit, for a fixed value of $\left|\mu_{0}\right| /(\hbar \omega) \gg 1$ [17. In this limit, a 1D classical field treatment is sufficient, with a Hamiltonian

$$
\mathcal{H}=\int_{\mathbb{R}} d x\left[-\frac{\hbar^{2}}{2 m} \psi^{*} \partial_{x}^{2} \psi+\frac{g}{2} \psi^{* 2} \psi^{2}+\frac{1}{2} m \omega^{2}(t) x^{2} \psi^{*} \psi\right] .
$$

We have slightly generalized (58) to treat the case of a time dependent trap:

$$
\omega^{2}(t)=\omega^{2} \chi(t)
$$

where the function $\chi(t)$, going from unity for $t=0$ to zero for $t \rightarrow+\infty$ describes the switch-off procedure of the trap. The atom number is fixed so that the norm squared of the classical field is also fixed:

$$
\int_{\mathbb{R}} d x|\psi(x)|^{2}=N
$$

This classical field problem can be solved on a computer, which will allow a test of our predictions for $N_{\text {exc }} / N$.

The problem can be further simplified in the limit $\hbar \omega /\left|\mu_{0}\right| \rightarrow 0$, where one expects that the field $\psi(x)$ will remain "close" (up to a phase factor) to the one describing a pure soliton [18], $\psi_{0}(x)=N^{1 / 2} \phi_{0}(x)$, with

$$
\phi_{0}(x)=\frac{1}{2 \xi^{1 / 2}} \frac{1}{\cosh [x /(2 \xi)]} .
$$

We then use the number conserving Bogoliubov formalism of [19,20], downgraded to a classical field problem (simply replacing commutators with Poisson brackets), as was already done in 21]. One splits the field by projection along the mode $\phi_{0}$ and orthogonally to it:

$$
\psi(x)=a_{0} \phi_{0}(x)+\psi_{\perp}(x)
$$

where $a_{0}$ is the component of the field on the mode $\phi_{0}$ and the field $\psi_{\perp}(x)$ is orthogonal to that mode. The idea is to treat $\psi_{\perp}(x)$ as a small perturbation. The strength of the number conserving approach is to eliminate the amplitude $a_{0}$ in a systematic way, using the modulus-phase representation

$$
a_{0}=\left|a_{0}\right| e^{i \theta} \text {. }
$$

The phase $\theta$ is eliminated by a redefinition of the transverse field:

$$
\Lambda(x) \equiv e^{-i \theta} \psi_{\perp}(x) .
$$

The modulus $\left|a_{0}\right|$ is expressed in terms of $\Lambda$ using the condition of a fixed atom number (62).

In the absence of trapping potential, one keeps terms up to quadratic in $\Lambda$ in the Hamiltonian, and the resulting quadratic form can be written in normal form as 22

$$
\mathcal{H}_{0} \simeq \mathcal{E}_{0}+\frac{P^{2}}{2 m N}+\int_{\mathbb{R}} \frac{d k}{2 \pi} \epsilon_{k} b_{k}^{*} b_{k}
$$


where $\mathcal{E}_{0}=N \mu_{0} / 3$ is the ground state of the classical field model. The variable $P$ of the field represents the total momentum of the field, written to first order in $\Lambda$ :

$$
P=\frac{\hbar}{i} N^{1 / 2} \int_{\mathbb{R}} d x \phi_{0}^{\prime}(x)\left[\Lambda^{*}(x)-\Lambda(x)\right] .
$$

The occurrence of the term $P^{2} /(2 m N)$ represents physically the fact that the center of mass motion is decoupled; more formally, it corresponds to the fact that $\phi_{0}(x)$ "spontaneously" breaks the translational symmetry of the Hamiltonian, which leads to the occurrence of a Goldstone mode [23, 24, 25]. The field variable canonically conjugated to $P$ corresponds to the center of mass position of the field, written up to first order in $\Lambda$ :

$$
Q=N^{-1 / 2} \int_{\mathbb{R}} d x x \phi_{0}(x)\left[\Lambda(x)+\Lambda^{*}(x)\right] .
$$

Apart from this Goldstone mode, the other eigenmodes behave as a continuum of decoupled harmonic oscillators, with normal (complex) variables $b_{k}$ and eigenenergies

$$
\epsilon_{k}=\left|\mu_{0}\right|+\frac{\hbar^{2} k^{2}}{2 m}
$$

which correspond to internal (and thus gapped) excitations of the gas: An elementary excitation physically takes the form of a free particle coming from infinity with a wavevector $k$ and scattering on a soliton with $N-1$ particles. The field variable $b_{k}$ has the expression

$$
b_{k} \equiv \int_{\mathbb{R}} d x\left[u_{k}^{*}(x) \Lambda(x)-v_{k}^{*}(x) \Lambda^{*}(x)\right]
$$

where the Bogoliubov modes of the number conserving theory are expressed as follows in Dirac's notation (see 20] $\S \mathrm{V} . \mathrm{A})$ here for a real function $\phi_{0}$ :

$$
\begin{aligned}
\left|u_{k}\right\rangle & =\mathcal{Q}\left|U_{k}\right\rangle \\
\left|v_{k}\right\rangle & =\mathcal{Q}\left|V_{k}\right\rangle
\end{aligned}
$$

where $\mathcal{Q}=1-\left|\phi_{0}\right\rangle\left\langle\phi_{0}\right|$ projects orthogonally to $\left|\phi_{0}\right\rangle$, and the $U_{k}, V_{k}$ are the eigenmodes of the usual Bogoliubov-de Gennes equations

$$
\begin{aligned}
\epsilon_{k} U_{k}(x)= & {\left[-\frac{\hbar^{2}}{2 m} \partial_{x}^{2}+2 g N\left|\phi_{0}(x)\right|^{2}-\mu_{0}\right] U_{k}(x) } \\
& +g N \phi_{0}^{2}(x) V_{k}(x) \\
-\epsilon_{k} V_{k}(x)= & {\left[-\frac{\hbar^{2}}{2 m} \partial_{x}^{2}+2 g N\left|\phi_{0}(x)\right|^{2}-\mu_{0}\right] V_{k}(x) } \\
& +g N \phi_{0}^{* 2}(x) U_{k}(x) .
\end{aligned}
$$

It turns out that these modes are known exactly [26]. For $k>0$ one has

$$
\begin{aligned}
& U_{k}(x)=e^{i K X} \frac{1+\left(K^{2}-1\right) \cosh ^{2} X+2 i K \sinh X \cosh X}{(K-i)^{2} \cosh ^{2} X} \\
& V_{k}(x)=\frac{e^{i K X}}{(K-i)^{2} \cosh ^{2} X}
\end{aligned}
$$

where (differently from section 3) we have set $X=x /(2 \xi)$ and $K=2 k \xi$. The modes for $k<0$ are deduced from the relations $U_{k}(x)=U_{-k}(-x)$ and $V_{k}(x)=V_{-k}(-x)$.

In presence of the trap, there is an extra contribution to the Hamiltonian containing the trapping energy,

$$
\mathcal{W}=\frac{1}{2} m \omega^{2}(t) \int_{\mathbb{R}} d x x^{2} \psi^{*}(x) \psi(x) .
$$

We approximate it to leading non-trivial order in $\Lambda$, that is to first order, injecting the splitting (64). The term in $a_{0}^{*} a_{0}$ deviates from $N$ by $O\left(\Lambda^{2}\right)$ so it gives to first order only a constant contribution, with an integral over $x$ that can be calculated exactly if necessary. The crossed terms $a_{0}^{*} \Lambda$ and complex conjugate are kept, with $a_{0}$ approximated by $N^{1 / 2} e^{i \theta}$, whereas the quadratic terms $\Lambda^{*} \Lambda$ are neglected. Finally, we replace the field $\Lambda(x)$ by its modal expansion

$$
\begin{aligned}
\Lambda(x)=-N^{1 / 2} \phi_{0}^{\prime}(x) & Q+\frac{i}{\hbar N^{1 / 2}} x \phi_{0}(x) P \\
& +\int_{\mathbb{R}} \frac{d k}{2 \pi}\left[u_{k}(x) b_{k}+v_{k}^{*}(x) b_{k}^{*}\right] .
\end{aligned}
$$

We thus keep

$\mathcal{W} \simeq \frac{1}{2} N m \omega^{2}(t) \int_{\mathbb{R}} d x x^{2} \phi_{0}^{2}(x)+\chi(t) \int_{\mathbb{R}} \frac{d k}{2 \pi}\left[\gamma_{k} b_{k}+\gamma_{k}^{*} b_{k}^{*}\right]$,

with coefficients

$$
\gamma_{k}=N^{1 / 2} \frac{1}{2} m \omega^{2} \int_{\mathbb{R}} d x x^{2} \phi_{0}(x)\left[u_{k}(x)+v_{k}(x)\right] .
$$

With the residues method, the resulting integral can be evaluated analytically. We give for convenience the ratio to the mode eigenenergy:

$$
\frac{\gamma_{k}}{\epsilon_{k}}=-\left(\frac{\hbar \omega}{\mu_{0}}\right)^{2} \frac{\pi(N \xi)^{1 / 2} / 2}{(K-i)^{2}\left(1+K^{2}\right)} \frac{1}{\cosh (K \pi / 2)}
$$

where $K=2 k \xi$ as above.

When the perturbation $\mathcal{W}$ is added to the unperturbed Hamiltonian (67), the equilibrium value of the $b_{k}$ 's minimizing the resulting energy is

$$
b_{k}(0)=-\frac{\gamma_{k}^{*}}{\epsilon_{k}}
$$

At later times, the trap is opened according to the switchoff function $\chi(t)$. The Hamiltonian equations of motion then give

$$
i \hbar \frac{d}{d t} b_{k}(t)=\epsilon_{k} b_{k}(t)+\chi(t) \gamma_{k}^{*},
$$

to be solved with the initial conditions (83):

$$
b_{k}(t)=b_{k}(0)\left[\chi(t)-e^{-i \omega_{k} t} \int_{0}^{t} d \tau e^{i \omega_{k} \tau} \frac{d \chi(\tau)}{d \tau}\right]
$$

where $\omega_{k}=\epsilon_{k} / \hbar$ is the mode frequency. This allows to calculate the number of excitations after the trap was witched off, using $\chi(t) \rightarrow 0$ for $t \rightarrow+\infty$ :

$$
N_{\mathrm{exc}} \equiv \lim _{t \rightarrow+\infty} \int_{\mathbb{R}} \frac{d k}{2 \pi} b_{k}^{*}(t) b_{k}(t)=\int_{\mathbb{R}} \frac{d k}{2 \pi}\left|b_{k}(0)\right|^{2} I\left(\omega_{k}\right)
$$


where $I(\Omega)$ is a spectral density of the switch-off procedure at frequency $\Omega$ :

$$
I(\Omega)=\left|\int_{0}^{+\infty} d \tau e^{-i \Omega \tau} \frac{d \chi(\tau)}{d \tau}\right|^{2} .
$$

We recall that (86) is valid up to leading order in $N$ (because of the classical field model) and to leading order in $\omega^{2}$ (because of the perturbative treatment of the deviations of the field from the free space soliton).

The case producing the maximal number of excitations for a monotonic $\chi(t)$ corresponds to a sudden trap switchoff, where $I(\Omega)=1$ at all frequencies. Using (82), we find that the resulting integral can be evaluated with the residues method, so that

$$
N_{\mathrm{exc}}^{\mathrm{sud}}=C N\left(\frac{\hbar \omega}{\mu_{0}}\right)^{4}
$$

with

$$
\begin{aligned}
C & =\frac{\pi}{16} \int_{\mathbb{R}} d K \frac{1}{\left(1+K^{2}\right)^{4}} \frac{1}{\cosh ^{2}(K \pi / 2)} \\
& =\frac{\pi^{2}\left(\pi^{2}+25\right)}{3840}+\frac{\zeta(5)+5 \zeta(3)}{128}=0.1446785 \ldots
\end{aligned}
$$

This result is encouraging since a moderately small value $\hbar \omega=\left|\mu_{0}\right| / 10$ already leads to a number of excitations relative to the total atom number at the $10^{-5}$ level. As shown in Fig 2 the analytical prediction (88) is in good agreement with the number of excitations deduced from a numerical solution of the Gross-Pitaevskii equation, provided that $\left|\mu_{0}\right| \gg \hbar \omega$.

The number of excitations can be reduced by switching off the trap more slowly. E.g. a linear ramping

$$
\chi(t)=(1-\lambda t) \theta(1-\lambda t)
$$

leads to

$$
I(\Omega)=4\left(\frac{\lambda}{\Omega}\right)^{2} \sin ^{2}[\Omega /(2 \lambda)] .
$$

Due to the presence of a gap $\left|\mu_{0}\right|$ in the internal excitation spectrum of the gas, one gets the upper bound on the number of excitations

$$
N_{\mathrm{exc}} \leq\left(\frac{2 \hbar \lambda}{\mu_{0}}\right)^{2} N_{\mathrm{exc}}^{\mathrm{sud}}
$$

that is one gains quadratically with the switch-off time when it becomes longer than the internal soliton time $\hbar /\left|\mu_{0}\right|$.

\section{Conclusion}

Inspired by recent observations of matter wave bright solitons in atomic gases, we have considered here two problems that may be relevant for experiments.

The first problem is strictly beyond mean field: It corresponds to the pair correlations between positions of the

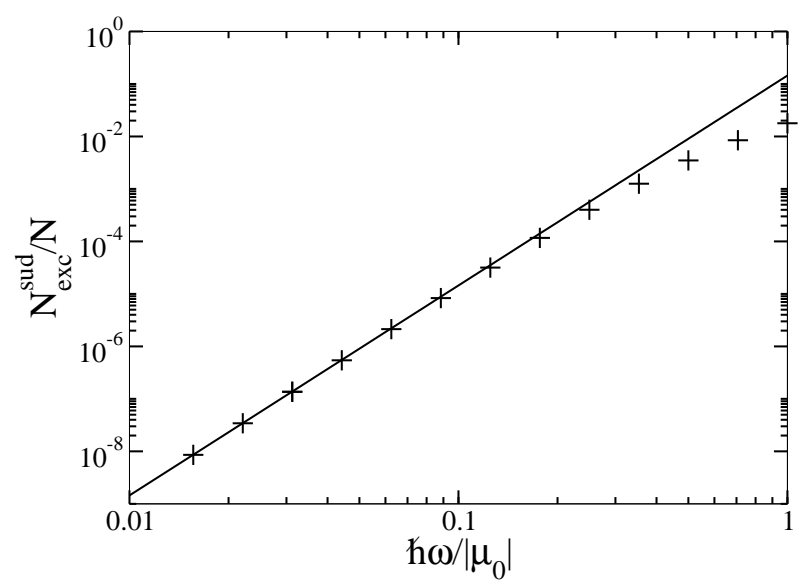

Fig. 2. For an initially harmonically trapped classical soliton, number of excitations $N_{\text {exc }}^{\text {sud }}$ produced by a sudden opening of the trap, divided by the number of particles $N$, and given as a function of $\hbar \omega /\left|\mu_{0}\right|$, in log-log scale. Solid line: Analytical result (88) obtained in the limit $\left|\mu_{0}\right| \gg \hbar \omega$. Symbols: Result deduced from a numerical solution of the Gross-Pitaevskii equation in the trap. Here $\omega$ is the oscillation frequency of the particles in the trap, and $\mu_{0}=-m g^{2} N^{2} / 8 \hbar^{2}$ is the chemical potential that the classical soliton would have in the absence of trapping potential, $m$ being the particle mass and $g$ the coupling constant describing the interactions in 1D.

particles in a quantum soliton for a fixed position of the center of mass of the soliton. In particular, we have obtained analytically the large $N$ limit expression of these pair correlations, see (41) and (44). By integrating (41) over the center of mass position, we obtain a large $N$ expansion of the static structure factor for a fully delocalized center of mass position, a quantity already studied in 6 , 7. On an experimental point of view, our predictions can be tested by measuring the positions of the particles in a very broad quantum soliton, prepared with weak attractive interactions and a relatively small atom number 27 .

The second problem was studied in the classical field model. The number of internal excitations of the gas created by the trap opening from an initial pure soliton was calculated in the limit where the soliton size is smaller than the size of the harmonic single particle ground state, see (88) for a sudden trap opening. This also can be seen experimentally by detecting atoms flying away from the remaining soliton core after the trap opening. A possible extension of this calculation is to include quantum fluctuations of the field.

We acknowledge useful discussions with L. Khaykovich, C. Weiss, A. Sinatra, M. Olshanii. Our group is a member of IFRAF.

\section{A Calculation of the Fourier transforms of $\rho(x \mid 0)$ and $\rho(x, y \mid 0)$}

We start with the definition of the mean density for a fixed center of mass position $R$, taking here $R=0$ without loss 
of generality: From (7) one has

$$
\begin{array}{r}
\rho(x \mid 0)=\int_{\mathbb{R}^{N}} d x_{1} \ldots d x_{N} \delta\left(\sum_{k=1}^{N} x_{k} / N\right)\left[\sum_{j=1}^{N} \delta\left(x_{j}-x\right)\right] \\
\times\left|\phi\left(x_{1}, \ldots, x_{N}\right)\right|^{2} .
\end{array}
$$

Using the bosonic exchange symmetry we can restrict the integral to the fundamental domain $D$ of (8), including a factor $N$ !. For simplicity we take in this appendix $\hbar^{2} /(m|g|)$ as the unit of length. With the change of variables, of Jacobian equal to unity,

$$
x_{j}=\sum_{k=1}^{j} u_{k}, \quad 1 \leq j \leq N,
$$

the condition to be in $D$ is simply that all the $u_{2}, \ldots, u_{N}$ are positive, and $u_{1}$ can vary in the whole real space $\mathbb{R}$. Setting

$$
\beta_{k}=\sum_{j=k}^{N}[2 j-(N+1)]=(N+1-k)(k-1),
$$

we obtain from (9):

$$
\begin{array}{r}
\rho(x \mid 0)=[(N-1) !]^{2} \sum_{j=1}^{N} \int_{\mathbb{R}} d u_{1} \int_{\left(\mathbb{R}^{+}\right)^{N-1}} d u_{2} \ldots d u_{N} \\
\delta\left(\sum_{k=1}^{N} \frac{N+1-k}{N} u_{k}\right) \delta\left(x-\sum_{k=1}^{j} u_{k}\right) e^{-\sum_{k=2}^{N} \beta_{k} u_{k}} .
\end{array}
$$

One can calculate the integral over $u_{1}$ : The first delta factor in (96), the one ensuring that $R=0$, imposes a value

$$
u_{1}=-\sum_{k=2}^{N} \frac{(N+1-k)}{N} u_{k} .
$$

When one reports this value of $u_{1}$ in the argument of the second delta factor in (96), one obtains a remaining factor $\delta\left(x-\sum_{k=2}^{N} \alpha_{k, j} u_{k}\right)$, with

$$
\begin{aligned}
\alpha_{k, j} & =\frac{k-1}{N} \quad \text { for } k \leq j \\
& =-\frac{N+1-k}{N} \quad \text { for } k>j .
\end{aligned}
$$

Taking the Fourier transform of this remaining delta factor, according to $\tilde{\rho}\left(q_{a} \mid 0\right)=\int_{\mathbb{R}} d x e^{-i q_{a} x} \rho(x \mid 0)$, gives

$$
\begin{aligned}
\tilde{\rho}\left(q_{a} \mid 0\right)= & {[(N-1) !]^{2} \sum_{j=1}^{N} \int_{\left(\mathbb{R}^{+}\right)^{N-1}} d u_{2} \ldots d u_{N} } \\
& \times e^{-i q_{a} \sum_{k=2}^{N} \alpha_{k, j} u_{k}} e^{-\sum_{k=2}^{N} \beta_{k} u_{k}} \\
= & \sum_{j=1}^{N} \prod_{k=2}^{N} \frac{\beta_{k}}{\beta_{k}+i q_{a} \alpha_{k, j}}
\end{aligned}
$$

where we used the fact that the product of all $\beta_{k}$ (for $k$ from 2 to $N)$ is equal to $[(N-1) !]^{2}$. Replacing the $\alpha_{k, j}$ by their expression and using the identity

$$
\prod_{k=j}^{N-1}(k+z)=\frac{\Gamma(N+z)}{\Gamma(j+z)} \quad \forall j \in 1, \ldots, N
$$

deduced from the basic property $\Gamma(z+1)=z \Gamma(z)$ of the Gamma function, with the convention that an "empty" product is equal to unity, one gets (18).

We now turn to the pair distribution function for a center of mass position fixed in $R=0$. Following the same steps as for the mean density, we obtain the Fourier transform

$$
\tilde{\rho}\left(q_{a}, q_{b} \mid 0\right)=\sum_{1 \leq j \neq k \leq N} \prod_{n=2}^{N} \frac{\beta_{n}}{\beta_{n}+i q_{a} \alpha_{n, j}+i q_{b} \alpha_{n, k}} .
$$

We can restrict to a summation over $j<k$, the contribution for $j>k$ being deduced by exchanging $q_{a}$ and $q_{b}$. In the product over $n$, three ranges have then to be considered, (i) the first range $n \leq j$, (ii) the mid-range $j+1 \leq n \leq k$, and (iii) the last range $k+1 \leq n \leq N$. The first range and the last range contributions can be expressed in terms of the Gamma function as in (102). The mid-range contribution is equal to the product

$$
P_{\text {mid }}=\prod_{n^{\prime}=j}^{k-1} \frac{\left(N-n^{\prime}\right) n^{\prime}}{\left(N-n^{\prime}\right) n^{\prime}-i Q_{a}\left(N-n^{\prime}\right)+i Q_{b} n^{\prime}}
$$

where we have reindexed the product setting $n^{\prime}=n-1$ and we have defined $Q_{a}=q_{a} / N$ and $Q_{b}=q_{b} / N$. The last step is to consider the denominator in each factor of (104) as a polynomial of degree 2 in $n^{\prime}$ : Its roots are $\epsilon_{a}$ defined in (16. 17) and $N+i\left(Q_{a}+Q_{b}\right)-\epsilon_{a}$. This leads to

$$
P_{\text {mid }}=\prod_{n^{\prime}=j}^{k-1} \frac{\left(N-n^{\prime}\right) n^{\prime}}{\left(n^{\prime}-\epsilon_{a}\right)\left[N+i\left(Q_{a}+Q_{b}\right)-\epsilon_{a}-n^{\prime}\right]}
$$

which can now be expressed as a ratio of products of Gamma functions. Then one gets (15).

\section{B Calculation of the variance of $\mathrm{O}_{2}$}

We explain how to calculate exactly the moments $\left\langle O_{2}^{n}\right\rangle_{0}$ of the quantity $\mathrm{O}_{2}$ defined in (32) in the $\mathrm{N}$-body internal ground state for a fixed center of mass position $R=0$. We take $\hbar^{2} / m|g|$ as unit of length and we use the transformations exposed at the beginning of appendix $\mathrm{A}$. Considering the change of variable (94), we rewrite $\mathrm{O}_{2}$ as

$$
\begin{aligned}
O_{2}= & \sum_{i=1}^{N}\left[\left(x_{i}-u_{1}\right)+u_{1}\right]^{2} \\
& =-N u_{1}^{2}+2 u_{1}\left(\sum_{i=1}^{N} x_{i}\right)+\sum_{i=1}^{N}\left(x_{i}-u_{1}\right)^{2} .
\end{aligned}
$$


The first sum in the right hand side of (106) will have a vanishing contribution, since the expectation value is taken for a zero center of mass position. Replacing $x_{i}-u_{1}$ by its expression in terms of $u_{2}, \ldots, u_{N}$, and using the fact that the value of $u_{1}$ is fixed to (97) we see that one may effectively replace $\mathrm{O}_{2}$ by the quantity

$$
\begin{array}{r}
O_{2} \rightarrow \sum_{i=2}^{N}\left(\sum_{k=2}^{i} u_{k}\right)^{2}-\frac{1}{N}\left(\sum_{k=2}^{N}(N+1-k) u_{k}\right)^{2} \\
\equiv \sum_{i, j=2}^{N} A_{i j} u_{i} u_{j}
\end{array}
$$

with the symmetric matrix

$$
A_{i j}=\frac{1}{N}[N+1-\max (i, j)][\min (i, j)-1] .
$$

We have thus reduced the problem to the calculation of the integrals

$$
\begin{aligned}
\left\langle O_{2}^{n}\right\rangle_{0}=[(N-1) !]^{2} \int_{\left(\mathbb{R}^{+}\right)^{N-1}} d u_{2} \ldots d u_{N} \\
\times\left(\sum_{i, j=2}^{N} A_{i j} u_{i} u_{j}\right)^{n} e^{-\sum_{k=2}^{N} \beta_{k} u_{k}}
\end{aligned}
$$

with $\beta_{k}$ defined in (95). These integrals may be calculated by interpreting them as Gaussian averages, introducing the auxiliary complex random variables $\alpha_{k}, 2 \leq k \leq N$ : These variables $\alpha_{k}$ are statistically independent and each one has a Gaussian probability distribution $\propto e^{-\left|\alpha_{k}\right|^{2} \beta_{k}}$. Each $u_{k}$ then corresponds to $\left|\alpha_{k}\right|^{2}$, so that

$$
\left\langle O_{2}^{n}\right\rangle_{0}=\left\langle\left\langle\left(\sum_{i, j=2}^{N} A_{i j}\left|\alpha_{i}\right|^{2}\left|\alpha_{j}\right|^{2}\right)^{n}\right\rangle\right\rangle .
$$

Here $\langle\langle\ldots\rangle\rangle$ denotes the Gaussian average of the $\alpha_{k}$ 's and can be calculated using Wick's theorem. The calculations are a bit lengthy for $n=2$ since Wick's theorem has to be applied to a product of 8 variables. Clearly the matrix $A_{i j} /\left(\beta_{i} \beta_{j}\right)$ appears, which is the matrix $B_{i j}$ of (38). We finally get (34) for $\left(\operatorname{Var} O_{2}\right)_{0}=\left\langle O_{2}^{2}\right\rangle_{0}-\left(\left\langle O_{2}\right\rangle_{0}\right)^{2}$.

\section{Large $N$ asymptotics of}

$\rho(x, y \mid 0)-\rho(x \mid 0) \rho(y \mid 0)$

To perform the large $N$ expansion we shall use the Fourier space expressions (15]18). We thus define

$$
\delta \tilde{\rho}\left(q_{a}, q_{b} \mid 0\right) \equiv \tilde{\rho}\left(q_{a}, q_{b} \mid 0\right)-\tilde{\rho}\left(q_{a} \mid 0\right) \tilde{\rho}\left(q_{b} \mid 0\right) .
$$

The pair distribution function involves a double sum over $j \neq k$. Since $\tilde{\rho}(q \mid 0)$ is a simple sum, the last term in (111) is a double sum over $j$ and $k$, without the restriction $j \neq$ $k$. It thus makes sense to split $\tilde{\rho}\left(q_{a} \mid 0\right) \tilde{\rho}\left(q_{b} \mid 0\right)$ into a offdiagonal part $(j \neq k)$, that we collect with the double sum in $\tilde{\rho}\left(q_{a}, q_{b} \mid 0\right)$, and a diagonal part

$$
\begin{aligned}
\operatorname{Diag}=\sum_{j=1}^{N} & \prod_{\eta=a, b}\left|\frac{\Gamma(N)}{\Gamma\left(N+i Q_{\eta}\right)}\right|^{2} \\
& \times \frac{\Gamma\left(N+1+i Q_{\eta}-j\right) \Gamma\left(j-i Q_{\eta}\right)}{\Gamma(N+1-j) \Gamma(j)},
\end{aligned}
$$

where $Q_{a, b}$ are defined above (16).

The idea to obtain the large $N$ limit is simply to replace the discrete sums by integrals. To this end, one has to calculate the large $N$ limit of each term of the sums, for fixed values of $y_{a} \equiv j / N$ and $y_{b} \equiv k / N$. The expansion of the Gamma functions is conveniently performed using

$\frac{\Gamma(z+a)}{\Gamma(z+b)}=e^{(a-b) \ln z}\left[1+\frac{(a-b)(a+b-1)}{2 z}+O\left(1 / z^{2}\right)\right]$

where the real quantity $z$ tends to $+\infty$, and the fixed quantities $a$ and $b$ may be complex. One also uses the large $N$ expansion of the quantity $\epsilon_{a}$ :

$$
\epsilon_{a}=i Q_{a}+\frac{Q_{a} Q_{b}}{N}+\ldots
$$

For the diagonal part, only the leading term of (113) is useful. Replacing $\sum_{j}$ by $N \int d y_{a}$ leads to

$$
\operatorname{Diag} \simeq N \int_{0}^{1} d y_{a} e^{i Q \ln \frac{1-y_{a}}{y_{a}}}=N \int_{\mathbb{R}} d X_{a} \frac{e^{i Q X_{a}}}{\left(2 \cosh \frac{X_{a}}{2}\right)^{2}}
$$

where $Q=Q_{a}+Q_{b}$ and the integral was transformed with the change of variable $X_{a}=\ln \frac{x_{a}}{1-x_{a}}$, to acquire the form of a Fourier transform. Note that the resulting integral can be calculated exactly, giving $\pi Q / \sinh (\pi Q)$, but this is not useful here.

For the double sum over $j<k$, one has to include the $1 / z$ term in the expansion (113), to obtain a non-zero result:

$$
\begin{gathered}
\text { Off-Diag } \simeq N Q_{a} Q_{b} \int_{0}^{1} d y_{a} \int_{y_{a}}^{1} d y_{b} e^{i Q_{a} \ln \frac{1-y_{a}}{y_{a}}} e^{i Q_{b} \ln \frac{1-y_{b}}{y_{b}}} \\
\times\left[2+\ln \frac{1-y_{a}}{y_{a}}-\ln \frac{1-y_{b}}{y_{b}}-\left(\frac{1}{y_{b}}+\frac{1}{1-y_{a}}\right)\right]+Q_{a} \leftrightarrow Q_{b} .
\end{gathered}
$$

The change of variables $X_{a}=\ln \frac{y_{a}}{1-y_{a}}$ and $X_{b}=\ln \frac{y_{b}}{1-y_{b}}$ gives to the off-diagonal contribution the form of a Fourier transform:

$$
\begin{aligned}
& \text { Off-Diag } \simeq N Q_{a} Q_{b} \int_{\mathbb{R}^{2}} d X_{a} d X_{b} \frac{e^{-i Q_{a} X_{a}} e^{-i Q_{b} X_{b}}}{\left(2 \cosh \frac{X_{a}}{2}\right)^{2}\left(2 \cosh \frac{X_{b}}{2}\right)^{2}} \\
& \times \theta\left(X_{b}-X_{a}\right)\left[X_{b}-X_{a}-e^{X_{a}}-e^{-X_{b}}\right]+Q_{a} \leftrightarrow Q_{b} .
\end{aligned}
$$

The leading term of $\delta \tilde{\rho}\left(q_{a}, q_{b} \mid 0\right)$ for $N \rightarrow+\infty$ for a fixed $\xi$ is the sum of (115) and (117). The Fourier transform with respect to $q_{a, b}$ is straightforward: The factors 
$Q_{a, b}$ act as derivatives, and the remaining bits have already a Fourier form. The Fourier transform of the diagonal contribution gives a contribution involving a factor $\delta\left(x_{a}-x_{b}\right)$, which exactly cancels with the first term in the right-hand side of (24), at the considered order in $N$, see (42). We obtain (41).

\section{References}

1. Toshiya Kinoshita, Trevor Wenger, David S. Weiss, Science 305, 1125 (2004); Zoran Hadzibabic, Peter Krüger, Marc Cheneau, Baptiste Battelier and Jean Dalibard, Nature 441, 1118 (2006).

2. S. Inouye, M.R. Andrews, J. Stenger, H.-J. Miesner, D.M. Stamper-Kurn, W. Ketterle, Nature 392, 151 (1998); S. L. Cornish, N. R. Claussen, J. L. Roberts, E. A. Cornell, and C. E. Wieman, Phys. Rev. Lett. 85, 1795 (2000).

3. L. Khaykovich, F. Schreck, G. Ferrari, T. Bourdel, J. Cubizolles, L. Carr, Y. Castin, C. Salomon, Science 296, 1290 (2002).

4. K. Strecker, G. Partridge, A. Truscott, R. Hulet, Nature 417, 150 (2002).

5. I. Mazets, G. Kurizki, Europhys. Lett. 76, 196 (2006).

6. P. Calabrese, J.-S. Caux, Phys. Rev. Lett. 98, 150403 (2007).

7. P. Calabrese, J.-S. Caux, J. Stat. Mech. P08032 (2007).

8. M. Olshanii, Phys. Rev. Lett. 81, 938 (1998).

9. J.B. Mc Guire, J. Math. Phys. 5, 622 (1964).

10. M. Gaudin, La fonction d'onde de Bethe (Masson, Paris, 1983).

11. Y. Castin, C. Herzog, Comptes Rendus de l'Académie des Sciences de Paris, tome 2, série IV, 419-443 (2001).

12. W. Ketterle, D.S. Durfee, and D.M. Stamper-Kurn, "Making, probing and understanding Bose-Einstein condensates", in Bose-Einstein condensation in atomic gases, Proceedings of the International School of Physics "Enrico Fermi", Course CXL, edited by M. Inguscio, S. Stringari and C.E. Wieman (IOS Press, Amsterdam, 1999), pp. 67176 .

13. F. Calogero and A. Degasparis, Phys. Rev. A 11, 265 (1975)

14. The calculation was done independently by Christopher Herzog [11, since the result of 13] was at that time unknown to the authors of 11 .

15. We also used the property $\frac{d}{d X} F(X, X)=2 \partial_{X} F(X, X)$ where $F(X, Y)$ is the function over which $\partial_{X} \partial_{Y}$ acts in the right hand side of (45).

16. One performs the change of variable $Y=X+Z$ in the integral over $Y$, then one exchanges the order of the integration, integrating first over $X$ (which can be done after the change of variable $\left.u=e^{X}\right)$. One is left with the integral over $\mathbb{R}$ of $Z(Z+2) /[\exp (Z)-1]$, which is readily expressed in terms of the Zeta function after a series expansion of $1 /[\exp (Z)-1]$ in powers of $\exp (-Z)$.

17. One may wonder, for a fixed $\left|\mu_{0}\right| /(\hbar \omega)$, how large $N$ should be to enter the asymptotic regime. In the absence of a detailed analysis, we give here a naive answer: When one uses Bogoliubov theory for the quantum field in presence of the trap, one finds that the ground Bogoliubov mode has an energy exactly equal to $\hbar \omega$, with mode functions $u(x)=[\hbar /(2 m \omega)]^{1 / 2}\left[(m \omega / \hbar) x \phi(x)-\phi^{\prime}(x)\right]$ and $v(x)=[\hbar /(2 m \omega)]^{1 / 2}\left[-(m \omega / \hbar) x \phi^{*}(x)-\phi^{\prime *}(x)\right]$, where $\phi(x)$ is the Gross-Pitaevskii condensate wavefunction normalized to unity and $\phi^{\prime}(x)$ is its derivative. This mode corresponds to the center of mass oscillation. It contributes to the number of non-condensed particles as $\int_{\mathbb{R}} d x|v(x)|^{2}$, scaling as $\left|\mu_{0}\right| /(\hbar \omega)$ in the small $\omega$ limit. The naive requirement is thus $\left|\mu_{0}\right| /(\hbar \omega) \ll N$.

18. There is an abundant literature about mean-field solitons. Restricting to the cold atom context, see e.g. the recent works and references therein: A. D. Martin, C. S. Adams, and S. A. Gardiner, Phys. Rev. A 77, 013620 (2008); C. Lee, J. Brand, Europhys. Lett. 73, 321 (2006); A. Gammal, L. Tomio, T. Frederico, Phys. Rev. A 66, 043619 (2002); L. Carr, Y. Castin, Phys. Rev. A 66, 063602 (2002).

19. C. Gardiner, Phys. Rev. A 56, 1414 (1997).

20. Y. Castin, R. Dum, Phys. Rev. A 57, 3008 (1998).

21. A. Sinatra, Y. Castin, E. Witkowska, Phys. Rev. A 75, 033616 (2007).

22. M. Olshanii (unpublished).

23. J.-P. Blaizot and G. Ripka, Quantum Theory of Finite Systems, The MIT Press (Cambridge, Massachusetts, 1986).

24. M. Lewenstein, Li You, Phys. Rev. Lett. 77, 3489 (1996).

25. Kanamoto R., Saito H. and Ueda M., Phys. Rev. Lett. 94, 090404 (2005).

26. D.J. Kaup, Phys. Rev. A 42, 5689 (1990).

27. Let us assume that the wavefunction $\Phi(R)$ of the center of mass of the gas is a Gaussian wavepacket of width $\sigma$, centered in $R=0$. To obtain in this case a pair correlation $\rho(x, y)-\rho(x) \rho(y)$ close to the one calculated here for a center of mass perfectly localized in $R=0$, we estimate that the condition $\sigma<\xi / \sqrt{N}$ should be satisfied, from the expansion $\rho(x) \simeq \rho(x \mid 0)+\frac{1}{2}\left\langle R^{2}\right\rangle\left[\partial_{R}^{2} \rho(x \mid R)\right]_{R=0}$ and $\rho(x, y) \simeq \rho(x, y \mid 0)+\frac{1}{2}\left\langle R^{2}\right\rangle\left[\partial_{R}^{2} \rho(x, y \mid R)\right]_{R=0}$, where $\left\langle R^{2}\right\rangle$ is the expectation value in the wavefunction $\Phi(R)$. Starting with a harmonically trapped gas in its internal plus center of mass ground state, as discussed in 28], one may change the scattering length after trap opening to adiabatically increase the soliton size, in order to decrease $\sqrt{N} \sigma / \xi$.

28. C. Weiss, Y. Castin, arXiv:0806.3395 (2008). 